\title{
Spin-Glass Ground State in a Triangular-Lattice Compound $\mathrm{YbZnGaO}_{4}$
}

\author{
Zhen Ma, ${ }^{1}$ Jinghui Wang, ${ }^{1}$ Zhao-Yang Dong, ${ }^{1}$ Jun Zhang, ${ }^{2}$ Shichao Li, ${ }^{1}$ Shu-Han Zheng, ${ }^{1}$ Yunjie Yu, ${ }^{2}$ Wei Wang, \\ Liqiang Che, ${ }^{3}$ Kejing Ran, ${ }^{1}$ Song Bao, ${ }^{1}$ Zhengwei Cai, ${ }^{1}$ P. Čermák, ${ }^{4}$ A. Schneidewind, ${ }^{4}$ S. Yano, ${ }^{5}$ J. S. Gardner, ${ }^{5,6}$ \\ Xin Lu, ${ }^{3,7}$ Shun-Li Yu, ${ }^{1,7, *}$ Jun-Ming Liu, ${ }^{1,7}$ Shiyan Li, ${ }^{2,7, \dagger}$ Jian-Xin Li, ${ }^{1,7, \$}$ and Jinsheng Wen ${ }^{1,7, \$}$ \\ ${ }^{1}$ National Laboratory of Solid State Microstructures and Department of Physics, Nanjing University, Nanjing 210093, China \\ ${ }^{2}$ State Key Laboratory of Surface Physics, Department of Physics, and Laboratory of Advanced Materials, \\ Fudan University, Shanghai 200433, China \\ ${ }^{3}$ Center for Correlated Matter and Department of Physics, Zhejiang University, Hangzhou 310058, China \\ ${ }^{4}$ Jülich Centre for Neutron Science (JCNS) at Heinz Maier-Leibnitz Zentrum (MLZ), \\ Forschungszentrum Jülich GmbH, Lichtenbergstr. 1, 85748 Garching, Germany \\ ${ }^{5}$ National Synchrotron Radiation Research Center, Hsinchu 30077, Taiwan \\ ${ }^{6}$ Center for Condensed Matter Sciences, National Taiwan University, Taipei 10617, Taiwan \\ ${ }^{7}$ Collaborative Innovation Center of Advanced Microstructures, Nanjing University, Nanjing 210093, China
}

(Received 30 November 2017; revised manuscript received 12 January 2018; published 22 February 2018)

We report on comprehensive results identifying the ground state of a triangular-lattice structured $\mathrm{YbZnGaO}_{4}$ as a spin glass, including no long-range magnetic order, prominent broad excitation continua, and the absence of magnetic thermal conductivity. More crucially, from the ultralow-temperature ac susceptibility measurements, we unambiguously observe frequency-dependent peaks around $0.1 \mathrm{~K}$, indicating the spin-glass ground state. We suggest this conclusion holds also for its sister compound $\mathrm{YbMgGaO}_{4}$, which is confirmed by the observation of spin freezing at low temperatures. We consider disorder and frustration to be the main driving force for the spin-glass phase.

DOI: 10.1103/PhysRevLett.120.087201

Quantum spin liquids (QSLs) represent a novel state of matter in which spins are highly entangled, but neither order nor freeze at low temperatures [1,2]. There is accumulating experimental evidence suggesting that such a state is realized in $\mathrm{YbMgGaO}_{4}$ [3-11]. The magnetic specific heat $C_{m}$ is proportional to $T^{\alpha}$ with $\alpha \approx 2 / 3[3,5,12]$. It has a negative Curie-Weiss temperature of $\Theta \sim-4 \mathrm{~K}$ $[3,4]$ but does not show a long-range magnetic order at low temperatures [5,6]. Moreover, diffusive continuous magnetic excitations have been observed by inelastic neutron scattering (INS) measurements [5,6], which are interpreted as resulting from the fractional spin excitations of a QSL $[13,14]$. However, there are also reports challenging this idea: (i) the thermal conductivity $\kappa$ study in Ref. [12] reveals no contributions to $\kappa$ from magnetic excitations despite the large magnetic specific heat at low temperatures, casting doubts on the existence of itinerant quasiparticles expected for a QSL [15]; (ii) since $\mathrm{Mg}^{2+}$ and $\mathrm{Ga}^{3+}$ in the nonmagnetic layers are randomly distributed $[3,4,16]$, the disorder effect, which is detrimental to the QSL phase for this compound [17], can be significant [5,8].

In this Letter, we report comprehensive measurements on a closely related system, $\mathrm{YbZnGaO}_{4}$. We show that the most natural conclusion that is consistent with the microand macroscopic data presented here is that the system is a spin glass. We suggest this conclusion to be also true for $\mathrm{YbMgGaO}_{4}$, further supported by the observation of spin freezing at low temperatures. We believe disorder $[5,8,11,16,17]$ and frustration $[4,5,11,18-22]$ to be largely responsible for this phase.

High-quality single crystals of $\mathrm{YbZnGaO}_{4}$ were grown by the floating-zone technique, overcoming the problem caused by the volatile nature of $\mathrm{ZnO}[3,23]$. The dc and ac magnetic susceptibility and specific heat were measured in a Quantum Design physical property measurement system (see the Supplemental Material [24] for details). INS experiments on the single crystals were carried out on PANDA located at MLZ at Garching, Germany [27]. In the measurements, the 11 coaligned single crystals weighed $1.2 \mathrm{~g}$ in total with a sample mosaic of $0.98^{\circ}$ and were mounted in the $(H, K, 0)$ plane. INS experiments on the 14-g powder sample were carried out on SIKA located at ANSTO at Lucas Heights, Australia. The wave vector $\boldsymbol{Q}$ was expressed as the $(H, K, L)$ reciprocal lattice unit (r.l.u.) of $\left(a^{*}, b^{*}, c^{*}\right)=(4 \pi / \sqrt{3} a, 4 \pi / \sqrt{3} b, 2 \pi / c)$ with $a=b=$ 3.414(2) $\AA$ and $c=25.140(2) \AA$.

$\mathrm{YbZnGaO}_{4}$ is isostructural to $\mathrm{YbMgGaO}_{4}$, both of which have the $\mathrm{YbFe}_{2} \mathrm{O}_{4}$-type structure (space group $R \overline{3} \mathrm{~m}$, No. 166) $[23,28,29]$. Schematics of the crystal structure and the two-dimensional triangular lattice of $\mathrm{Yb}^{3+}$ are illustrated in Figs. 1(a) and 1(b), respectively. The magnetic ground state of $\mathrm{Yb}^{3+}$ ions is a spin-1/2 Kramers doublet [see Fig. 1(d) or Refs. [3,5,8] ]. In $\mathrm{YbZnGaO}_{4}$, the dc magnetic susceptibility of the effective spin follows the Curie-Weiss 


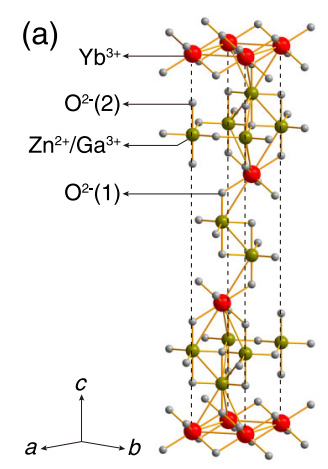

(b)

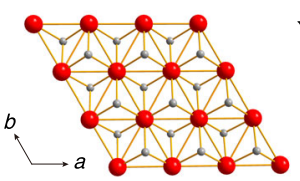

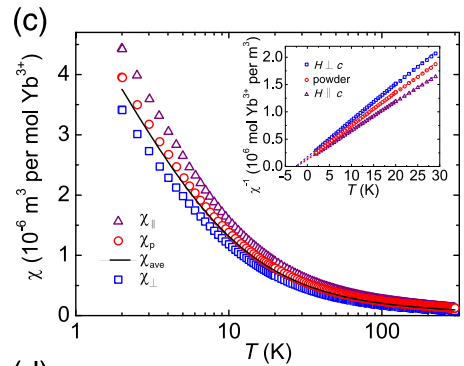

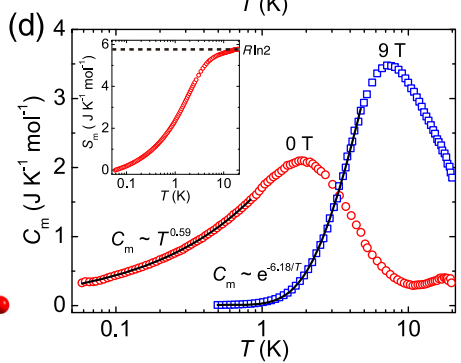

FIG. 1. (a) Schematic crystal structure for $\mathrm{YbZnGaO}_{4}$ and $\mathrm{YbMgGaO}_{4}$. (b) Top view of the triangular layer of $\mathrm{YbO}_{6}$ octahedra. (c) dc magnetic susceptibility for fields applied parallel $\left(\chi_{\|}\right)$and perpendicular $\left(\chi_{\perp}\right)$ to the $c$ axis for the single crystal, and polycrystalline sample $\left(\chi_{p}\right)$, measured with a $0.1-\mathrm{T}$ field. The data have been corrected by the Van Vleck paramagnetic susceptibility, as discussed in Ref. [24]. The solid line is the averaged susceptibility $\chi_{\text {ave }}$. The inset shows the inverse susceptibility and the accompanying Curie-Weiss fits. (d) Magnetic specific heat $C_{m}$ measured at zero and 9-T fields. $C_{m}$ is obtained by subtracting the contribution from the lattice using a nonmagnetic reference sample $\mathrm{LuZnGaO}_{4}$. Solid lines are fits to the data described in the main text. The inset shows the zero-field magnetic entropy $S_{m}$, obtained using $S_{m}=\int_{0}^{T} C_{m} / T d T$. The dashed line indicates $R \ln 2$ ( $R=$ the ideal gas constant).

law from 2 to $30 \mathrm{~K}$. In the inset of Fig. 1(c), we show the inverse susceptibility and the Curie-Weiss fits up to $30 \mathrm{~K}$. From the fits, we find $\Theta$ to be $-2.70(2),-2.38(3)$, and $-2.46(2) \mathrm{K}$, for the single crystal with magnetic fields perpendicular and parallel to the $c$ axis and for the polycrystalline sample, respectively. The negative sign shows

that the magnetic ground state is dominated by antiferromagnetic interactions. The superexchange coupling constant $J$ is estimated to be $1.73(5) \mathrm{K}$. These parameters are summarized in Table I, together with other values for this material, in comparison with $\mathrm{YbMgGaO}_{4}$.

In Fig. 1(d) we plot the magnetic specific heat $C_{m}$ down to $0.05 \mathrm{~K}$ for $\mathrm{YbZnGaO}_{4}$. From the zero-field data, we do not observe a $\lambda$-type peak expected for a well-defined phase transition. Instead, there is a broad peak at $T^{+} \approx 1.86(5) \mathrm{K}$, below which $C_{m}$ decreases. Below $T^{+}$, we fit $C_{m}$ to $T^{\alpha}$ and determine $\alpha$ to be 0.59 (2). We have also attempted to fit the low-temperature data using $C_{m} \sim \exp (-\Delta / T)$, and obtained a small gap of $0.05(3) \mathrm{K}$, consistent with the large magnetic specific heat arising from the gapless magnetic excitations at low temperatures. With increasing fields, $T^{+}$gets higher, and the hump becomes narrower. At $9 \mathrm{~T}, T^{+}$should correspond to a transition from the paramagnetic to the ferromagnetic state, as the system is in a fully polarized state at low temperatures [see Fig. S2(a)]. When we fit the 9-T data with $C_{m} \sim \exp (-\Delta / T)$, we obtain $\Delta=6.18(3) \mathrm{K}$, which corresponds to a magnon gap induced by an external magnetic field, as also observed in $\mathrm{YbMgGaO}_{4}$ [5,12]. In the inset of Fig. 1(d), we show that the magnetic entropy $S_{m}$ is precisely $R \ln 2$ ( $R=$ the ideal gas constant) at $20 \mathrm{~K}$, expected for a Kramers doublet in the ground state [3-5].

We now explore the system by carrying out INS experiments, which reveal similar behaviors to $\mathrm{YbMgGaO}_{4}[5,6]$. In Figs. 2(a) and 2(b), we present the contour maps of the excitation spectra at energy transfers of $E=0.3$ and $0.6 \mathrm{meV}$, respectively. The broad diffusive excitations spreading along edges of the two-dimensional (2D) Brilouin zone, and decreasing in intensity with increasing $Q$, indicate that the system is dominated by antiferromagnetic correlations but without long-range order, consistent with the macroscopic results in Figs. 1(c) and 1(d). Magnetic dispersions along two high-symmetry paths are plotted in Fig. 2(c), which exhibit a continuum over the whole energy range measured. The excitations are gapless (see also Fig. S4 [24]), consistent with the specific heat

TABLE I. Some parameters for $\mathrm{YbZnGaO}_{4}$ and $\mathrm{YbMgGaO}_{4}$.

\begin{tabular}{lccccccccccccc}
\hline \hline Compound & $\Theta_{\perp}(\mathrm{K})$ & $\Theta_{\|}(\mathrm{K})$ & $\Theta_{p}(\mathrm{~K})$ & $J(\mathrm{~K})$ & $g_{\perp}$ & $g_{\|}$ & $g_{p}$ & $T^{+}(\mathrm{K})$ & $\alpha$ & $\Delta(\mathrm{K})$ & $T_{f}(\mathrm{~K})$ & $\Delta P$ \\
\hline $\mathrm{YbZnGaO}_{4}$ & $-2.70(2)$ & $-2.38(3)$ & $-2.46(2)$ & $1.73(5)$ & $3.17(4)$ & $3.82(2)$ & $3.58(3)$ & $1.86(5)$ & $0.59(2)$ & $6.18(3)$ & $0.093(6)$ & $0.053(2)$ \\
$\mathrm{YbMgGaO}_{4}$ & -4.78 & -3.20 & -4.11 & $1.5^{\mathrm{a}}$ & 3.00 & 3.82 & 3.21 & 2.40 & 0.74 & 8.26 & $0.099(6)$ & $0.068(4)$ \\
\hline \hline
\end{tabular}

$\overline{\Theta_{\perp}, \Theta_{\|} \text {, and } \Theta_{p} \text { are the Curie-Weiss temperatures for the single crystal with magnetic fields perpendicular and parallel to the } c \text { axis, and }}$ for the polycrystalline sample, respectively. $J$ is the superexchange coupling constant, approximated by $[4] J=\left(4 J_{ \pm}+J_{z z}\right) / 3$, where $J_{ \pm} \approx-\Theta_{\perp} / 3=0.90(1) \mathrm{K}$, and $J_{z z} \approx-2 \Theta_{\|} / 3=1.59(2) \mathrm{K} . g_{\perp}, g_{\|}$, and $g_{p}$ are the Landé $g$ factors, obtained by fitting the magnetization data in Fig. S2 [24]. $T^{+}$is the peak temperature of the zero-field magnetic specific heat. $\alpha$ is the fitted index using $C_{m} \sim T^{\alpha} . \Delta$ is the magnon gap obtained by fitting the 9-T data with $C_{m} \sim \exp (-\Delta / T)$. Corresponding values for $\mathrm{YbMgGaO}_{4}$ are from Refs. $[3,4,6,12]$.

$T_{f}$ is the peak temperature of the real part of the ac susceptibility $\chi^{\prime}$ at $100 \mathrm{~Hz} . \Delta P$ is the peak shift $\left\{\left(\Delta T_{f}\right) /\left[T_{f} \Delta \lg (f)\right]\right\}$ from 100 to $10000 \mathrm{~Hz}$. These two parameters for both $\mathrm{YbZnGaO}_{4}$ and $\mathrm{YbMgGaO}_{4}$ are obtained from our own measurements.

${ }^{a}$ Note that in our discussions, we take $J$ to be $2.83 \mathrm{~K}$ for $\mathrm{YbMgGaO}_{4}$, larger than the $1.5 \mathrm{~K}$ reported in Ref. [4]. The larger $J$ is obtained by calculating with the Curie-Weiss temperatures given in Ref. [6]. 
(a)

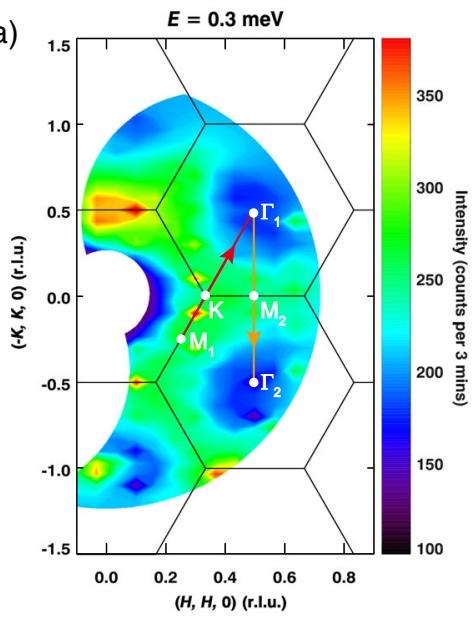

(b)

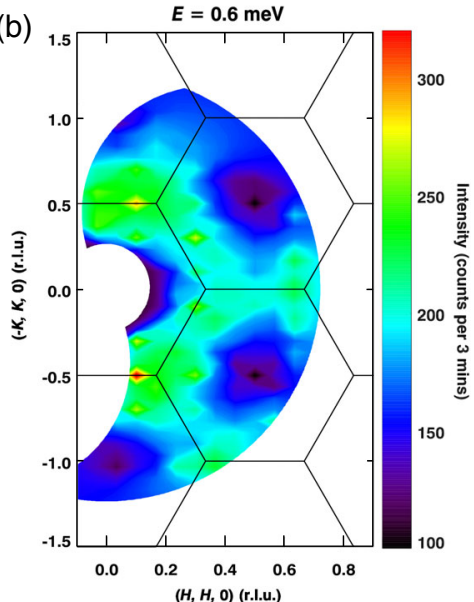

Intensity (counts per 3 mins)

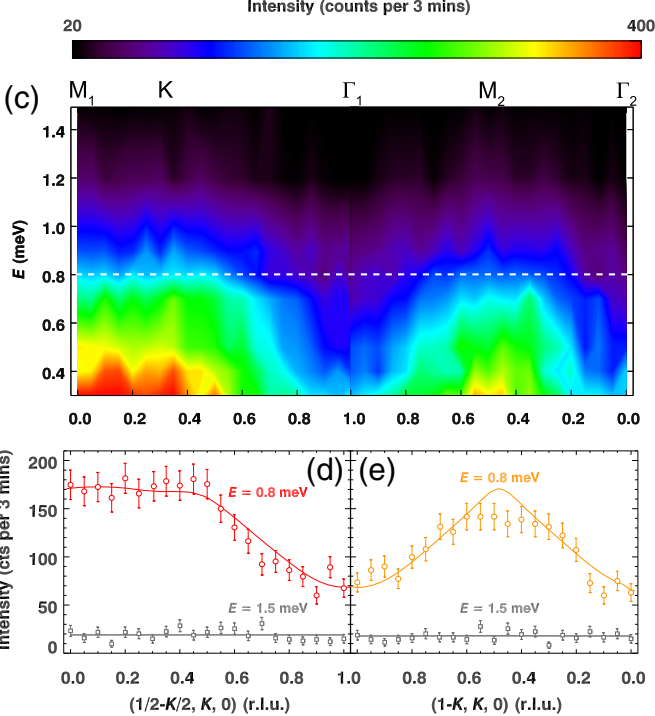

FIG. 2. (a) and (b) are contour maps of the INS spectra at $E=0.3$ and $0.6 \mathrm{meV}$, respectively, measured at $T=0.47 \mathrm{~K}$. The maps are obtained by plotting together a series of constant-energy scans along the $[H, 0,0]$ direction with a step size of 0.1 r.l.u., and an interval of 0.1 r.l.u. along the $[0, K, 0]$ direction. Solid lines indicate Brillouin zone boundaries. The additional bright feature around $(-0.5,0.5,0)$ in (a) does not represent a magnetic Bragg peak [24]. (c) Magnetic dispersion along the $M_{1}-K-\Gamma_{1}$ and $\Gamma_{1}-M_{2}-\Gamma_{2}$ directions as illustrated by the arrows in (a). The dispersion is obtained by plotting together a series of constant-energy scans as shown in (d) and (e), with an energy interval of $0.1 \mathrm{meV}$. The dashed line indicates constant-energy scans at $E=0.8 \mathrm{meV}$. In (d) and (e), lines through the data are the calculated results extracted from Fig. 3. Errors represent 1 standard deviation throughout the Letter.

data. Constant-energy scans along $M_{1}-K-\Gamma_{1}$ at two representative energies are shown in Fig. 2(d); similar scans along $\Gamma_{1}-M_{2}-\Gamma_{2}$ are shown in Fig. 2(e). At $E=0.8 \mathrm{meV}$, intensities remain roughly constant from $M_{1}$ to $(1 / 4,1 / 2,0)$, and then decrease as $Q$ approaches $\Gamma_{1}$. The scan along the $\Gamma_{1}-M_{2}-\Gamma_{2}$ direction results in a broad peak centering at $M_{2}$, and the spin-spin correlation length is estimated to be $3 \AA$ from this scan. This length scale is close to those obtained in $\mathrm{YbMgGaO}_{4}[5,6]$ and other QSL candidates [13,14].

As is shown in Refs. [5,6], a QSL phase can give rise to the observed INS spectra. However, we notice that the cations in the nonmagnetic layers are randomly distributed $[3,4,8,11,16]$. As a result, there should be a strong variation in the magnetic couplings due to the disordered charge environment $[8,17]$. In addition, the small $J$ will further exaggerate the disorder effect. Can disorder make the magnetic excitations mimic those expected for a QSL [17]? In this context, we consider introducing disorder into a stripe-order phase, which is suggested to be the ground state for $\mathrm{YbMgGaO}_{4}$ in the absence of disorder $[17,30]$. We use an anisotropic spin model with nearestneighbor and next-nearest-neighbor exchange interactions, which has been justified in Refs. [5,17], and perform calculations with the linear spin-wave theory [31]. Without disorder, gapless spin-wave excitations disperse up from the $M$ point. With increasing disorder, the welldefined spin-wave dispersions become broader both in momentum and energy. An example is presented in Fig. 3, and the calculated intensities are plotted together with the experimental data in Figs. 2(d) and 2(e). The calculated results agree with the experimental data quite well, demonstrating that an antiferromagnet with disorder can also exhibit the continuumlike INS spectra.

We further show thermal conductivity $\kappa$ results in Fig. 4(a). At $T=0.1 \mathrm{~K}, \kappa$ is only about half of that of the nonmagnetic sample $\mathrm{LuMgGaO}_{4}$ in which only phonons contribute to $\kappa$. This reduction is quite likely due to the scattering of phonons off the gapless magnetic excitations [12]. This also manifests itself in the magnetic-field measurements: in a field of $9 \mathrm{~T}$ that opens a gap of 6.18 (3) $\mathrm{K}$, there are almost no magnetic excitations to scatter phonons, so $\kappa$ increases. We fit the zero-field data with $\kappa / T=\kappa_{0} / T+n T^{\beta-1}$ up to $0.4 \mathrm{~K}$. Here, the first term $\kappa_{0}$ and
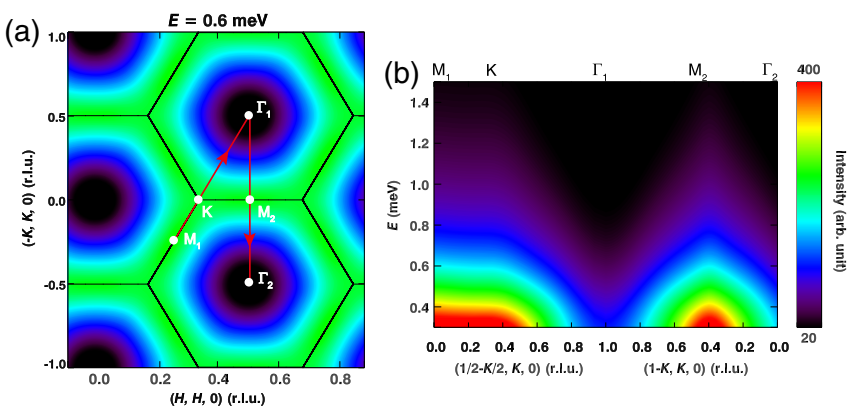

FIG. 3. (a) Contour map of the calculated spectra at $E=0.6 \mathrm{meV}$. (b) Calculated dispersions along the two highsymmetry paths illustrated in (a). 

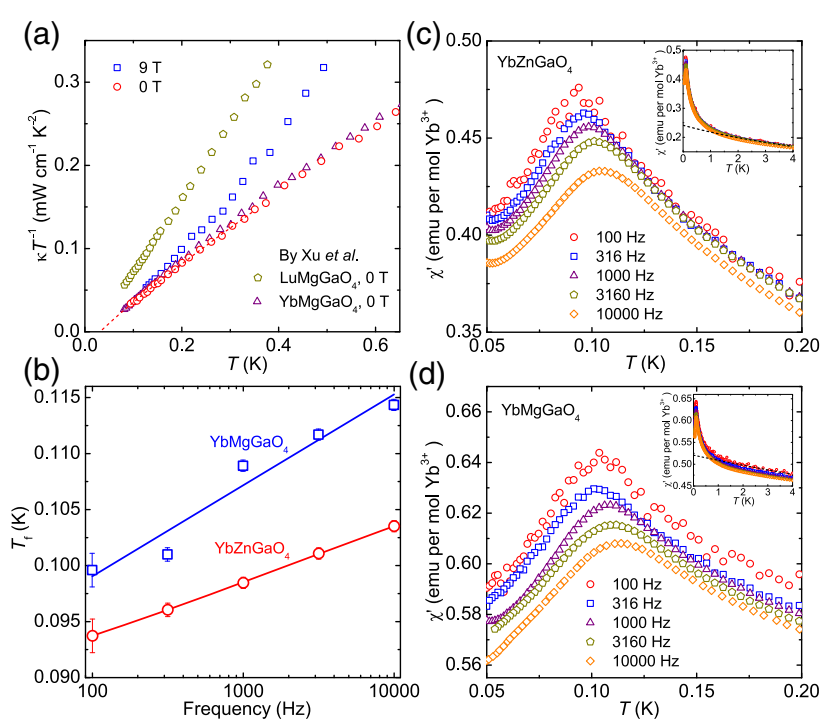

(d)

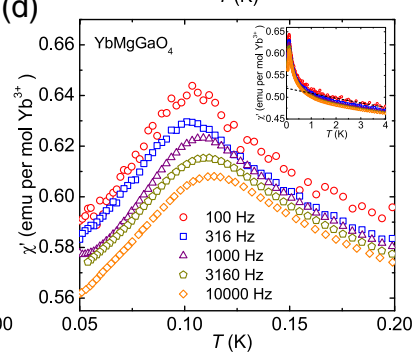

FIG. 4. (a) Thermal conductivity results on $\mathrm{YbZnGaO}_{4}$ under zero and 9-T magnetic fields applied parallel to the $c$ axis. The dashed line is a fit to the data described in the main text. For comparison, results on $\mathrm{YbMgGaO}_{4}$ and the nonmagnetic reference compound $\mathrm{LuMgGaO}_{4}$ are also plotted [12]. (b) Frequency dependence of the freezing temperature for both $\mathrm{YbZnGaO}_{4}$ and $\mathrm{YbMgGaO}_{4}$, extracted from the temperature dependence of the real part of the ac susceptibility $\chi^{\prime}$ shown in (c) and (d). Lines through data are guides to the eye. In the insets of (c) and (d), $\chi^{\prime}$ in an extended temperature range up to $4 \mathrm{~K}$ is plotted. Dashed lines indicate the Curie-Weiss fits for the $100-\mathrm{Hz}$ data.

second term $n T^{\beta}$ represent nonphonon and phonon contributions, respectively. From the fit, we obtain $\kappa_{0} / T=-0.011(2) \mathrm{mW} \mathrm{K}^{-2} \mathrm{~cm}^{-1}$, and $\beta=1.97(2)$. In the nonmagnetic sample $\mathrm{LuMgGaO}_{4}$, it is shown that $\kappa_{0} / T=-0.007 \mathrm{~mW} \mathrm{~K}^{-2} \mathrm{~cm}^{-1}$, and $\beta=2.07$ [12]. In both materials, $\kappa_{0} / T$ is virtually zero, similar to the case of $\mathrm{YbMgGaO}_{4}$ [12]. In contrast, another QSL candidate $\mathrm{EtMe}_{3} \mathrm{Sb}\left[\mathrm{Pd}(\mathrm{dmit})_{2}\right]_{2}$ has a high $\kappa_{0} / T=2 \mathrm{~mW} \mathrm{~K}^{-2} \mathrm{~cm}^{-1}$, considered to be a signature of highly mobile quasiparticles in the QSL state [15]. We therefore believe that a gapless QSL is not an applicable description for $\mathrm{YbZnGaO}_{4}$, because its magnetic excitations will contribute to $\kappa$ $[32,33]$. On the other hand, the thermal conductivity results can be understood within a disordered-magnet picture, in which the mean-free path of the magnons is reduced with disorder, and they are not expected to conduct heat.

Taking all of the aforementioned observations into account, we believe that $\mathrm{YbZnGaO}_{4}$ is a spin glass, with frozen, short-range correlations below the freezing temperature $T_{f}$ [34-36]. Such a phase can be identified from the ac susceptibility. Thus, we perform the measurements with temperatures spanning about three decades, ranging from 0.05 to $4 \mathrm{~K}$. The results are shown in Fig. 4(c). At a measuring frequency of $100 \mathrm{~Hz}$, the real part of the susceptibility $\chi^{\prime}$ shows a broad peak at $T_{f} \approx 0.093(6) \mathrm{K}$. The peak height decreases, and the peak temperature increases, with increasing driving frequency $f$. The frequency dependence of $T_{f}$ is shown in Fig. 4(b). As a quantitative measure, $\Delta P=\left\{\left(\Delta T_{f}\right) /\left[T_{f} \Delta \lg (f)\right]\right\}$ is 0.053 (2) with $f$ changing from 100 to $10000 \mathrm{~Hz}$. This value is close to those observed in other insulating spin glasses [34-38]. The strong frequency dependence evidences a broad distribution of the spin relaxation times around $T_{f}$, typical of a spin glass [34-36].

We also measure the ac susceptibility for $\mathrm{YbMgGaO}_{4}$. As shown in Fig. 4(d), the behaviors are similar to those of $\mathrm{YbZnGaO}_{4}$, albeit with a slightly higher $T_{f}$ of $0.099(6) \mathrm{K}$ at $100 \mathrm{~Hz}$. For $\mathrm{YbMgGaO}_{4}$, the peak shift from 100 to $10000 \mathrm{~Hz}$ $\Delta P$ is 0.068 (4) [Fig. 4(b)], larger than that for $\mathrm{YbZnGaO}_{4}$. In the insets of Figs. 4(c) and 4(d), we plot $\chi^{\prime}$ in the whole temperature range measured. At high temperatures, it follows the Curie-Weiss law. Below $\sim 2 \mathrm{~K}$, it rises more rapidly with cooling. Remarkably, this temperature coincides with $T^{+}$, below which the magnetic specific heat decreases.

The spin-glass phase identified from the ac susceptibility is a natural ground state for $\mathrm{YbZnGaO}_{4}$. (i) Disorder and frustration, the two ingredients for a spin glass [34-36,39], are present and strong in such materials $[4,5,8,11$, 16-22,40,41]. (ii) A spin glass maintains short-range spin-spin correlations [34-36], consistent with the absence of a long-range magnetic order. (iii) The observed INS spectra can be nicely reproduced by bringing disorder into an ordered state. (iv) Macroscopically, a spin glass is disordered, and thus the magnons do not conduct heat due to the short mean-free path. This explains the lack of a contribution to the thermal conductivity from the gapless magnetic excitations. (v) Finally, we estimate the fraction of frozen moment to be 13(3)\% from our INS results shown in Fig. S4 [24], close to the $16(3) \%$ in $\mathrm{YbMgGaO}_{4}$ [5], but smaller than the $33 \%$ expected from theory [42]. We consider it to be a consequence of the strong frustration in this compound.

We also note that some findings in $\mathrm{YbZnGaO}_{4}$ suggest deviations from a generic spin glass. For instance, $T^{+}$is about 20 times $T_{f}$, much larger than that expected for a typical spin glass [34-36]. We believe that this indicates the existence of strong frustration. In addition to the geometrical frustration inherent in the triangular structure $[1,2]$, the spin-space anisotropy induced by the spin-orbit coupling of the $\mathrm{Yb}^{3+}$ ions, recognized in our anisotropic spin model and in Refs. [4,5,11,18-22], should further reduce $T_{f}$. Moreover, the strong disorder $[5,8,11,16,17]$ is expected to result in a reduced $T_{f}$. In some spin glasses, $C_{m} \sim T^{\alpha}$ with $\alpha=1$ at low temperatures $[43,44]$, but disorder may reduce this exponent [45].

In summary, we have successfully grown high-quality single crystals for $\mathrm{YbZnGaO}_{4}$, and our comprehensive measurements on these crystals provide concrete evidence that it is a spin glass. We show this conclusion is also applicable to $\mathrm{YbMgGaO}_{4}$. We suggest that the spin-glass phase in both compounds is driven by disorder and 
frustration. Our work reveals the very similar characteristics between QSL and spin-glass phases, including the broad "continuum" of magnetic excitations, and is a cautionary tale about labeling such materials without a full study of the spin system.

We acknowledge the Applications Group at Quantum Design for measuring the ac susceptibility. We thank Fengqi Song and Haijun $\mathrm{Bu}$ for help in measuring the high-field magnetization. We are grateful for stimulating discussions with Jia-Wei Mei, Shao-Chun Li, Weiqiang Yu, Lei Shu, D. Adroja, Guangyong Xu, and J. M. Tranquada. The work was supported by the National Natural Science Foundation of China with Grants No. 11374143, No. 11674157, No. 11774152, No. 11374138, No. 11674158, No. 11374257, and No. U1630248, and by the National Key Projects for Research \& Development of the Ministry of Science and Technology of China with Grants No. 2016YFA0300401, No. 2016YFA0300101, and No. 2016YFA0300503.

Z. M., J. H. W., Z.-Y. D., and J. Z. contributed equally to this work.

*slyu@nju.edu.cn

† shiyan_li@fudan.edu.cn

\#jxli@nju.edu.cn

jwen@nju.edu.cn

[1] P. Anderson, Mater. Res. Bull. 8, 153 (1973).

[2] L. Balents, Nature (London) 464, 199 (2010).

[3] Y. Li, H. Liao, Z. Zhang, S. Li, F. Jin, L. Ling, L. Zhang, Y. Zou, L. Pi, Z. Yang, J. Wang, Z. Wu, and Q. Zhang, Sci. Rep. 5, 16419 (2015).

[4] Y. Li, G. Chen, W. Tong, L. Pi, J. Liu, Z. Yang, X. Wang, and Q. Zhang, Phys. Rev. Lett. 115, 167203 (2015).

[5] J. A. M. Paddison, M. Daum, Z. Dun, G. Ehlers, Y. Liu, M. B. Stone, H. Zhou, and M. Mourigal, Nat. Phys. 13, 117 (2017).

[6] Y. Shen et al., Nature (London) 540, 559 (2016).

[7] Y. Li, D. Adroja, P. K. Biswas, P. J. Baker, Q. Zhang, J. Liu, A. A. Tsirlin, P. Gegenwart, and Q. Zhang, Phys. Rev. Lett. 117, 097201 (2016).

[8] Y. Li, D. Adroja, R. I. Bewley, D. Voneshen, A. A. Tsirlin, P. Gegenwart, and Q. Zhang, Phys. Rev. Lett. 118, 107202 (2017).

[9] Y. Li, D. Adroja, D. Voneshen, R. I. Bewley, Q. Zhang, A. A. Tsirlin, and P. Gegenwart, Nat. Commun. 8, 15814 (2017).

[10] Y. Shen, Y.-D. Li, H. C. Walker, P. Steffens, M. Boehm, X. Zhang, S. Shen, H. Wo, G. Chen, and J. Zhao, arXiv:1708.06655.

[11] X. Zhang, F. Mahmood, M. Daum, Z. Dun, J. A. M. Paddison, N. J. Laurita, T. Hong, H. Zhou, N. P. Armitage, and M. Mourigal, arXiv:1708.07503.

[12] Y. Xu, J. Zhang, Y. S. Li, Y. J. Yu, X. C. Hong, Q. M. Zhang, and S. Y. Li, Phys. Rev. Lett. 117, 267202 (2016).

[13] C. Balz et al., Nat. Phys. 12, 942 (2016).

[14] T.-H. Han, J. S. Helton, S. Chu, D. G. Nocera, J. A. Rodriguez-Rivera, C. Broholm, and Y.S. Lee, Nature (London) 492, 406 (2012).
[15] M. Yamashita, N. Nakata, Y. Senshu, M. Nagata, H. M. Yamamoto, R. Kato, T. Shibauchi, and Y. Matsuda, Science 328, 1246 (2010)..

[16] L. Balents, Nature (London) 540, 534 (2016).

[17] Z. Zhu, P. A. Maksimov, S. R. White, and A. L. Chernyshev, Phys. Rev. Lett. 119, 157201 (2017).

[18] Y.-D. Li, Y. Shen, Y. Li, J. Zhao, and G. Chen, arXiv:1608.06445.

[19] Y.-D. Li, Y.-M. Lu, and G. Chen, Phys. Rev. B 96, 054445 (2017).

[20] S. Tóth, K. Rolfs, A. R. Wildes, and C. Rüegg, arXiv:1705.05699.

[21] Y.-D. Li and G. Chen, Phys. Rev. B 96, 075105 (2017).

[22] Y.-D. Li, X. Wang, and G. Chen, Phys. Rev. B 94, 035107 (2016).

[23] N. Kimizuka and E. Takayama, J. Solid State Chem. 41, 166 (1982).

[24] See Supplemental Material at http://link.aps.org/ supplemental/10.1103/PhysRevLett.120.087201 for the detailed methodology, structure and element analyses, additional dc magnetization and specific heat results, and linear spin-wave calculations, which include Refs. [25,26].

[25] A. Amann, M. Nallaiyan, L. Montes, A. Wilson, and S. Spagna, IEEE Trans. Appl. Supercond. 27, 1 (2017).

[26] T. Holstein and H. Primakoff, Phys. Rev. 58, 1098 (1940).

[27] A. Schneidewind and P. Čermák, J. Large Scale Res. Facil. 1, A12 (2015).

[28] R. Cava, A. Ramirez, Q. Huang, and J. Krajewski, J. Solid State Chem. 140, 337 (1998).

[29] M. Orita, H. Tanji, M. Mizuno, H. Adachi, and I. Tanaka, Phys. Rev. B 61, 1811 (2000).

[30] Q. Luo, S. Hu, B. Xi, J. Zhao, and X. Wang, Phys. Rev. B 95, 165110 (2017).

[31] S. Toth and B. Lake, J. Phys. Condens. Matter 27, 166002 (2015).

[32] C. P. Nave and P. A. Lee, Phys. Rev. B 76, 235124 (2007).

[33] O. I. Motrunich, Phys. Rev. B 72, 045105 (2005).

[34] K. Binder and A. P. Young, Rev. Mod. Phys. 58, 801 (1986).

[35] J. A. Mydosh, Spin Glasses: An Experimental Introduction (Taylor \& Francis, London, Washington, DC, 1993).

[36] J. A. Mydosh, Hyperfine Interact. 31, 347 (1986).

[37] D. Hüser, L. E. Wenger, A. J. van Duyneveldt, and J. A. Mydosh, Phys. Rev. B 27, 3100 (1983).

[38] K. Mehlawat, G. Sharma, and Y. Singh, Phys. Rev. B 92, 134412 (2015).

[39] J. Wu, J. S. Wildeboer, F. Werner, A. Seidel, Z. Nussinov, and S. A. Solin, Europhys. Lett. 93, 67001 (2011).

[40] K. Fritsch, K. A. Ross, G. E. Granroth, G. Ehlers, H. M. L. Noad, H. A. Dabkowska, and B. D. Gaulin, Phys. Rev. B 96, 094414 (2017).

[41] S. Calder, S. R. Giblin, D. R. Parker, P. P. Deen, C. Ritter, J. R. Stewart, S. Rols, and T. Fennell, J. Phys. Condens. Matter 25, 356002 (2013).

[42] K. A. Ross, J. W. Krizan, J. A. Rodriguez-Rivera, R. J. Cava, and C. L. Broholm, Phys. Rev. B 93, 014433 (2016).

[43] D. Sherrington and S. Kirkpatrick, Phys. Rev. Lett. 35, 1792 (1975).

[44] A. Georges, O. Parcollet, and S. Sachdev, Phys. Rev. Lett. 85, 840 (2000).

[45] I. Kimchi, A. Nahum, and T. Senthil, arXiv:1710.06860. 